Pacific Journal of Mathematics

LEFSCHETZ FIXED POINT THEOREMS FOR A NEW CLASS 


\title{
LEFSCHETZ FIXED POINT THEOREMS FOR A NEW CLASS OF MULTI-VALUED MAPS
}

\author{
Michael J. Powers
}

The Lefschetz fixed point theorem states that whenever the Lefschetz number $\Lambda(f)$ of a map $f: X \rightarrow X$ is nonzero, then $f$ must have a fixed point. The theorem is known to hold when $X$ is an $A N R$ and $f$ is a compact continuous map. The theorem has been studied for compact, upper semi-continuous, acyclic multi-valued maps and is known to hold in this setting for topologically complete $A N R$ 's.

A more general class of multi-valued maps is considered in this paper: the class of compact upper semi-continuous maps which can be written as a composition of acyclic maps. Using this class of maps, a theorem is proved which generates spaces for which the Lefschetz theorem holds. In particular, the Lefschetz theorem holds for all (metric) $A N R$ 's.

Lefschetz fixed point theorems for multi-valued maps of compact spaces were first studied by Eilenberg and Montgomery [2]. Motivated by the LeRay-Schauder fixed point results, the concept of Lefschetz space has been generalized to that of $\Lambda$-space and $M \Lambda$ space for multi-valued maps ([3], [4], [6], [8]). A space $X$ is a $A$-space if, for every compact map $f: X \rightarrow X$, the Lefschetz number is defined and the Lefschetz theorem holds. An $M A$-space is defined in a similar way for multi-valued maps. The multi-valued maps, however, are required to be both compact and acyclic (i.e., the image of each point is an acyclic subset). This additional condition is necessary in order to define the induced homomorphism on the homology groups.

A method of generating $M \Lambda$-spaces from known $M \Lambda$-spaces was presented in [8]. In applying the method, however, the acyclicity condition created a problem. It was possible to conclude, for example, that (metric) $A N R$ 's where $\Lambda$-spaces, but not that such spaces were $M \Lambda$-spaces. While enlarging the concept of $M \Lambda$-space to include maps which are compositions of acyclic maps, a more general generating theorem can be proved. The category of all metric $A N R$ 's is produced by this generating theorem.

2. Definitions. The image of a subset $A$ of $X$ under a multivalued map $F: X \rightarrow Y$ is $F(A)=\bigcup_{\bigcup_{x A}} F(x)$. The map $F$ is compact if $F(X)$ is contained in a compact subset of $Y$. Let $\Gamma(F)$ denote the graph of $F$. The projections $p: \Gamma(F) \rightarrow X$ and $q: \Gamma(F) \rightarrow Y$ are 
called the projections associated with $F . F$ is upper semi-continuous (u.s.c.) if :

(i) $F(x)$ is compact for each $x \in X$ and

(ii) for each $x \in X$ and each open set $V$ containing $F(x)$, there is an open neighborhood $U$ of $x$ such that $F(U) \subset V$.

If $F: X \rightarrow Y$ and $G: Y \rightarrow Z$ are multi-valued maps, the composition of $F$ and $G$ is denoted $G \circ F: X \rightarrow Z$ and is defined by $G \circ F(x)=$ $\mathrm{U}_{y \in F(x)} G(y)$. The composition of u.s.c. maps is again u.s.c.

A point $x$ is a fixed point for $F: X \rightarrow X$ if $x \in F(x)$.

Eilenberg and Montgomery [2] defined an induced homomorphism for certain multi-valued maps $F: X \rightarrow Y$ with $X$ and $Y$ compact. Their definition was extended to the non-compact case in [8]. Only the basic definitions are given here. Let $\mathscr{T}$ denote the category of Hausdorff spaces and continuous maps. Let $\mathscr{A}$ denote the category of graded vector spaces over the field $Q$ of rational numbers and homomorphisms of degree zero. Let $\mathscr{H}: \mathscr{T} \rightarrow \mathscr{A}$ be a covariant functor which satisfies the homotopy axiom and agrees with the Čech homology functor $\check{H}$ on the subcategory of compact spaces. We also require that $\mathscr{H}$ satisfy a Vietoris mapping theorem of the following type:

If $f: X \rightarrow Y$ is a morphism in $\mathscr{T}$ such that

(i) $f$ is inverse acyclic (i.e., for each $y \in Y, f^{-1}(y)$ is acyclic with respect to $\mathscr{H}$ ) and

(ii) $f$ is proper (i.e., for each compact subset $K$ of $Y, f^{-1}(K)$ is compact), then $\mathscr{H}(f)$ is an isomorphism in $\mathscr{A}$.

A functor $\vec{H}$ satisfying these conditions was exhibited in [8].

A multi-valued map $F: X \rightarrow Y$ is acyclic (with respect to $\mathscr{H}$ ) if for each $x \in X, F(x)$ is an acyclic subset of $Y$. When $F: X \rightarrow Y$ is u.s.c. and acyclic the projection $p: \Gamma(F) \rightarrow X$ satisfies the conditions for the Vietoris Theorem above.

Definition 2.1. Let $F: X \rightarrow Y$ be an u.s.c. acyclic multivalued map of spaces in $\mathscr{T}$. The homomorphism induced by $F$ (with respect to $\mathscr{H}$ ) is defined $\mathscr{H}(F)=\mathscr{H}(q) \circ \mathscr{H}(p)^{-1}$, where $p, q$ are the projections associated with $F$. We write $\mathscr{C}(F)=F_{*}$.

Theorem 2.2. Let $F: X \rightarrow Y$ and $G: Y \rightarrow Z$ be u.s.c. acyclic maps of spaces in $\mathscr{T}$. If $G \circ F$ is acyclic, then $(G \circ F)_{*}=G_{*} \circ F_{*}$. (See [8], Theorem (3.8).)

Finally, we recall the definition of trace for endomorphisms of infinite dimensional vector spaces given by Leray [7]. Let $\phi$ be an 
endomorphism of a vector space $V$; let $N_{\phi}=\left\{v \mid \phi^{p}(v)=0\right.$ for some $p \geqq 1\}$. Suppose that $V / N_{\phi}$ has finite dimension. Then the trace of $\phi, \operatorname{tr}(\phi)$ or $\operatorname{tr}_{V}(\phi)$, is defined by $\operatorname{tr}_{V}(\phi)=\operatorname{tr}_{V / N_{\phi}}\left(\phi^{\prime}\right)$, where $\phi^{\prime}$ is induced by $\phi$ and $\operatorname{tr}\left(\phi^{\prime}\right)$ is the classical trace.

DeFinition 2.3. Let $\phi=\left\{\dot{\phi}_{k}\right\}: V \rightarrow V$ be an endomorphism (of degree zero) in $\mathscr{A}$. Then $f$ is said to be of finite type if

(i) $V_{k} / N_{\phi_{k}}$ has finite dimension for each $k$ and

(ii) $N_{\phi_{k}}=V_{k}$ in all but a finite number of dimensions $k$.

DeFINITION 2.4. Let $\phi: V \rightarrow V$ be an endomorphism of finite type in . A. The Lefschetz number of $\phi$ is

$$
\Lambda(\phi)=\sum_{k=0}^{\infty}(-1)^{k} \operatorname{tr}\left(\phi_{k}\right)
$$

Lemma 2.5. Let $\phi: V \rightarrow W$ and $\psi: W \rightarrow V$ be homomorphisms (of degree zero) in $\mathscr{A}$. If $\psi \circ \phi: V \rightarrow V$ is of finite type, then $\phi \circ \psi$ : $W \rightarrow W$ is also of finite type and in each dimension $k, \operatorname{tr}\left(\psi_{k}^{\circ} \phi_{k}\right)=$ $\operatorname{tr}\left(\phi_{k} \circ \psi_{k}\right)$. (See [7], Prop. c.)

3. $M \Lambda$-spaces. We will consider u.s.c. maps $F: X \rightarrow X$ which can be factored into a sequence $G_{n} \circ \ldots \circ G_{0}$ of acyclic u.s.c. maps. We will then want to use $\Lambda\left(G_{n^{*}} \circ \ldots \circ G_{0^{*}}\right)$ as a Lefschetz number for $F$. However, an easy example shows that some care must be exercised. It is possible to have

$$
G_{2} \circ G_{1}=F=H_{2^{\circ}} H_{1} \text { and } \Lambda\left(G_{2^{*}} \circ G_{1^{*}}\right) \neq 0, \Lambda\left(H_{2^{*}} \circ H_{1^{*}}\right)=0 \text {. }
$$

Simply let $F: S^{2} \rightarrow S^{2}$ by $F(x)=S^{2}$ for all $x \in S^{2}$. Define $K: S^{2} \rightarrow S^{2}$ by $K(x)=\left\{y \in S^{2} \mid\|x-y\| \leqq 7 / 4\right\}$. Then $K$ is u.s.c. and acyclic. In fact, $K$ is homotopic to the identity on $S^{2}$ and hence $K_{*}$ is the identity. Let $G_{1}=G_{2}=K$. Let $H_{1}$ be defined by $H_{1}(x)=K(-x)$

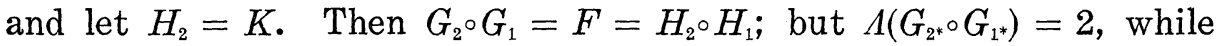
$\Lambda\left(H_{2^{*}} \circ H_{1^{*}}\right)=0$.

\section{$\mathscr{T}_{0}$ will denote a subcategory of $\mathscr{T}$.}

Definition 3.1. An u.s.c. map $F: X \rightarrow X$ of a space in $\mathscr{T}$ is admissible (relative to $\mathscr{T}_{0}$ ) if there are maps

$$
G_{i}: Y_{i} \longrightarrow Y_{i+1}, i=0, \cdots, n
$$

(where $Y_{0}=Y_{n+1}=X$ ) satisfying

(i) $F=G_{n} \circ \cdots \circ G_{0}$,

(ii) $G_{i}$ is acyclic and u.s.c. for each $i=0, \cdots, n$, and 
(iii) $Y_{i}$ is in $\mathscr{T}_{0}$, for $i=1, \cdots, n$.

Each such sequence $G_{0}, \cdots, G_{n}$ is called an admissible sequence for $F$.

Definition 3.2. An admissible map $F: X \rightarrow X$ is an $M$-Lefschetz map (relative to $\mathscr{T}_{0}$ ) if

(i) for each admissible sequence $G_{0}, \cdots, G_{n}$ for $F, G_{n^{*}} \circ \cdots \circ G_{1^{*}}$ has finite type and

(ii) whenever $G_{0}, \cdots, G_{n}$ is an admissible sequence with

$$
\Lambda\left(G_{n^{*}} \circ \cdots \circ G_{1^{*}}\right) \neq 0,
$$

then $F$ must have a fixed point.

Definition 3.3. A space $X$ is an $M$-Lefschetz space [ML-space] (relative to $\mathscr{T}_{0}$ ) if each admissible [compact admissible] map $F: X \rightarrow X$ (relative to $\mathscr{T}_{0}$ ) is an $M$-Lefschetz map (relative to $\mathscr{T}_{0}$ ).

Note that a single-valued continuous map $f$ is admissible iff $f_{*}$ has finite type. Thus the theory here is no more general in the case of single-valued maps than that presented in [6].

The concept of $M$-Lefschetz space and $M \Lambda$-space is more general than that presented in [8]. We continue to use the same terminology since the definition presented here seems to be the one that most warrants further study. Moreover, it will be shown that spaces already known to be $M$-Lefschetz or $M A$-spaces remain $M$-Lefschetz and $M \Lambda$-spaces in this more general setting.

4. Generating theorems. The following theorems present a method for generating $M \Lambda$-spaces from known $M \Lambda$-spaces. Similar theorems hold for $M$-Lefschetz spaces. It was recently pointed out to the author that this method of generating $M \Lambda$-spaces was used by W. Hurewitz in his lectures for the proof of the Lefschetz theorem for compact $A N R$ 's.

Definition 4.1. Let $F, G: X \rightarrow Y$ be multi-valued maps and $\alpha \in \operatorname{Cov} Y$, the set of open covers of $Y$. Then $F$ is $\alpha$-near $G$ if for each $x \in X$ and each $y \in F(x)$, there is a $U$ in $\alpha$ containing $y$ and meeting $G(x)$.

THeOREM 4.2. Let $X$ be a $T_{3}$-space in $\mathscr{T}_{0}$. Suppose that for each $\alpha \in \operatorname{Cov} X$ there is an $M \Lambda$-space $Y_{\alpha}$ (relative to $\mathscr{T}_{0}$ ) and maps $H_{\alpha}: X \rightarrow Y_{\alpha}, K_{\alpha}: Y_{\alpha} \rightarrow X$ satisfying

(i ) $H_{\alpha}, K_{\alpha}$ are u.s.c. and acyclic,

(ii) $K_{\alpha^{*}} \circ H_{\alpha^{*}}=1_{\mathscr{C}(X)}$, and 
(iii) $K_{\alpha} \circ H_{\alpha}$ is $\alpha$-near $1_{X}$.

Then $X$ is an MA-space(relative to $\left.\mathscr{T}_{0}\right)$.

Proof. Let $F: X \rightarrow X$ be an u.s.c. compact map which is admissible (relative to $\mathscr{T}_{0}$ ). Let $G_{0}, \cdots, G_{n}$ be an admissible sequence for $F$. Take any $\alpha \in \operatorname{Cov} X$ and $H_{\alpha}, K_{\alpha}, Y_{\alpha}$ as in the hypothesis. Consider the diagram

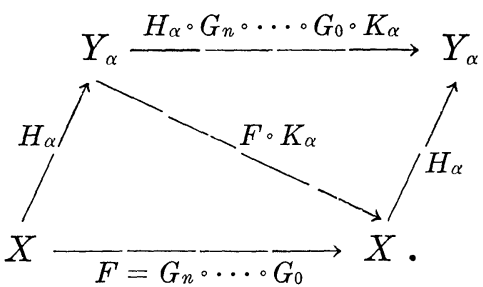

The map $H_{\alpha} \circ G_{n} \circ \cdots \circ G_{0} \circ K_{\alpha}$ is compact and admissible (relative to $\left.\mathscr{T}_{0}\right)$. Since $Y_{\alpha}$ is an $M \Lambda$-space $H_{\alpha^{*}} \circ\left(G_{n^{*}} \circ \ldots \circ G_{0^{*}} \circ K_{\alpha^{*}}\right)$ has finite type. Thus by (2.5) $\left(G_{n^{*}} \circ \cdots \circ G_{0^{*}} \circ K_{\alpha^{*}}\right) \circ H_{\alpha^{*}}=G_{n^{*}} \circ \cdots \circ G_{0^{*}}$ has finite type and $\Lambda\left(G_{n^{*}} \circ \cdots \circ G_{0^{*}}\right)=\Lambda\left(H_{\alpha^{*}} \circ G_{n^{*}} \circ \cdots \circ G_{0^{*}} \circ K_{\alpha^{*}}\right)$.

Now suppose $\Lambda\left(G_{n^{*}} \circ \cdots \circ G_{0^{*}}\right) \neq 0$. Then for each $\alpha \in \operatorname{Cov} X$, $H_{\alpha} \circ G_{n} \circ \ldots \circ G_{0} \circ K_{\alpha}$ is a compact admissible map and

$$
\Lambda\left(H_{\alpha^{*}} G_{n^{*}} \circ \cdots \circ G_{0^{*}} K_{\alpha^{*}}\right) \neq 0 .
$$

Thus the map has a fixed point $y_{\alpha} \in Y_{\alpha}$.

Since $y_{\alpha} \in H_{\alpha} \circ F \circ K_{\alpha}\left(y_{\alpha}\right)$, we can choose $\bar{x}_{\alpha}$ in $F\left(K_{\alpha}\left(y_{\alpha}\right)\right) \subseteq \overline{F(X)}$ such that $y_{\alpha} \in H_{\alpha}\left(\bar{x}_{\alpha}\right)$. Choose $x_{\alpha}$ in $K_{\alpha}\left(y_{\alpha}\right)$ such that $\bar{x}_{\alpha} \in F\left(x_{\alpha}\right)$. Finally, since $K_{\alpha} \circ H_{\alpha}$ is $\alpha$-near $1_{X}$ and since $x_{\alpha} \in K_{\alpha}\left(y_{\alpha}\right) \subseteq K_{\alpha} \circ H_{\alpha}\left(\bar{x}_{\alpha}\right)$, then there is an element $U_{\alpha}$ of $\alpha$ which contains both $x_{\alpha}$ and $\bar{x}_{\alpha}$. Now $\left\{\bar{x}_{\alpha}: \alpha \in \operatorname{Cov} X\right\}$ is a net in the compact space $\overline{F(X)}$ and there is a subnet $T=\{T(m): m \in E\}$ converging to a point $x_{0} \in \overline{F(X)} \subset X$. (Then there is a map $N: E \rightarrow \operatorname{Cov} X$ of directed sets satisfying $T(m)=\bar{x}_{N(m)}$ and for each $\alpha \in \operatorname{Cov} X$, there is an $n \in E$ such that $m>n$ implies $N(m)>\alpha$.)

Consider the net $S=\{S(m): m \in E\}$ defined by $S(m)=x_{N(m)}$. It suffices to prove that $S$ also converges to $x_{0}$. For then $S \times T$ is a net in $\Gamma(F)$ converging to $\left(x_{0}, x_{0}\right)$. But since $F$ is u.s.c. its graph is closed. Hence $\left(x_{0}, x_{0}\right) \in \Gamma(F)$ and $x_{0} \in F\left(x_{0}\right)$.

Let $V$ be any neighborhood of $x_{0}$. To show that $S$ is eventually in $V$, first recall that $x_{\alpha}$ and $\bar{x}_{\alpha}$ are both elements of $U_{\alpha}$ for each $\alpha$ in $\operatorname{Cov} X$. Since $X$ is regular, there is an open neighborhood $W$ of $x_{0}$ such that $\bar{W} \subset V$. Let $\alpha_{0}=\{V, X-\bar{W}\}$ in $\operatorname{Cov} X$. Since the net $T$ converges to $x_{0}$, there is an element $n_{W}$ in $E$ such that $m>n_{W}$ implies $T(m) \in W$. Moreover, there is an element $n_{0}$ of $E$ such that 
$N(m)>\alpha_{0}$ when $m>n_{0}$. Since $E$ is directed, there exists an element $n$ of $E$ such that $n>n_{0}, n_{W}$. Now for any $m>n$ it is easily verified that $S(m) \in V$. For $T(m)=\bar{x}_{N(m)} \in W$ and $\bar{x}_{N(m)}, x_{N(m)}$ are both in $U_{N(m)}$. Then $U_{N(m)} \cap W \neq \varnothing$ and hence $U_{N(m)} \not \subset X-\bar{W}$. Since $m>n_{0}, N(m)>\alpha_{0}$ and $U_{N(m)} \subset V$. Thus $x_{N(m)}=S(m) \in V$.

Condition (iii) of the theorem is fairly restrictive. However, for the present applications $H_{\alpha}$ and $K_{\alpha}$ will be single-valued maps and the condition is perfectly reasonable.

COROLLARY 4.3. A retract of an MA-space is again an $M \Lambda$-space.

If $X$ is a metric space, let $\left\{\varepsilon_{k}\right\}$ be a sequence of positive numbers converging to 0 and let $\alpha_{k}$ be some open cover of $X$ by $\varepsilon_{k}$-balls, for each $k$. Then while $D=\left\{\alpha_{k}\right\}$ is not cofinal in $\operatorname{Cov} X$ in general, we still obtain the following theorem.

THEOREM 4.4. Let $X$ be a metric space in $\mathscr{T}_{0}$. Suppose that for each $\alpha_{k} \in D$ there is an $M \Lambda$-space $Y_{k}$ (relative to $\left.\mathscr{T}_{0}\right)$ and maps $H_{k}$ : $X \rightarrow Y_{k}$ and $K_{k}: Y_{k} \rightarrow X$ satisfying

(i ) $H_{k}, K_{k}$ are u.s.c. and acyclic,

(ii) $K_{k^{*}} \circ H_{k^{*}}=1_{\mathscr{C}(X)}$, and

(iii) $K_{k} \circ H_{k}$ is $\varepsilon_{k}$-near $1_{X}$ (i.e., for each $\left.x \in X, K_{k} \circ H_{k}(x) \subset B\left(x ; \varepsilon_{k}\right)\right)$. Then $X$ is an $M \Lambda$-space (relative to $\mathscr{T}_{0}$ ).

Proof. Let $F: X \rightarrow X$ be an u.s.c. compact map which is admissible (relative to $\mathscr{T}_{0}$ ). Let $G_{0}, \cdots, G_{n}$ be an admissible sequence for $F$. Then for each integer $k$ and $H_{k}, K_{k}, Y_{k}$ as in the hypothesis it can be proved, just as in (4.2), that

$$
\Lambda\left(G_{n^{*}} \circ \cdots \circ G_{0^{*}}\right)=\Lambda\left(H_{k^{*}} \circ G_{n^{*}} \circ \cdots \circ G_{0^{*}} \circ K_{k^{*}}\right) .
$$

Suppose that $\Lambda\left(G_{n^{*}} \circ \cdots \circ G_{0^{*}}\right) \neq 0$. Then for each $k$, the compact admissible map $H_{k} \circ G_{n} \circ \cdots \circ G_{0} \circ K_{k}: Y_{k} \rightarrow Y_{k}$ has nonzero Lefschetz number and hence has a fixed point $y_{k} \in Y_{k}$. Just as in the proof of (4.2), $y_{k} \in H_{k} \circ F \circ K_{k}\left(y_{k}\right)$ and there are points $\bar{x}_{k}$ in $F\left(K_{k}\left(y_{k}\right)\right)$ and $x_{k}$ in $K_{k}\left(y_{k}\right)$ such that $y_{k} \in H_{k}\left(\bar{x}_{k}\right)$ and $\bar{x}_{k} \in F\left(x_{k}\right)$. Now since $K_{k} \circ H_{k}$ is $\varepsilon_{k^{-}}$ near $1_{X}$ and since $x_{k} \in K_{k}\left(y_{k}\right) \subset K_{k} \circ H_{k}\left(\bar{x}_{k}\right)$, then $d\left(x_{k}, \bar{x}_{k}\right)<\varepsilon_{k}$. Now $\left\{\bar{x}_{k}\right\}$ is a sequence in the compact space $\overline{F(X)}$ and there is a subsequence, still denoted $\left\{\bar{x}_{k}\right\}$, which converges to a point $x_{0} \in \overline{F(X)} \subset X$.

It suffices to prove that the corresponding subsequence of $\left\{x_{k}\right\}$ also converges to $x_{0}$. For then $\left\{\left(x_{k}, \bar{x}_{k}\right)\right\}$ is a sequence in $\Gamma(F)$ converging to $\left(x_{0}, x_{0}\right)$ and $x_{0} \in F\left(x_{0}\right)$. Given $\varepsilon>0$, choose $k_{1}$ so that for $k>k_{1}, \varepsilon_{k}<\varepsilon / 2$. Choose $k_{2}$ so that for $k>k_{2}, d\left(\bar{x}_{k}, x_{0}\right)<\varepsilon / 2$. Then for $k>\max \left\{k_{1}, k_{2}\right\}, d\left(x_{k}, \bar{x}_{k}\right)<\varepsilon_{k}<\varepsilon / 2$ and hence $d\left(x_{k}, x_{0}\right)<\varepsilon$. Then 
the subsequence $\left\{x_{k}\right\}$ converges to $x_{0}$.

5. Applications. In this section, $\mathscr{T}_{0}$ will be the category $\mathscr{T}_{M}$ of metric spaces and continuous maps.

Lemma 5.1. Let $f: X \rightarrow Y$ and $g: Y \rightarrow Z$ be maps in $\mathscr{T}$ which are proper and inverse acyclic (with respect to $\mathscr{\mathscr { C }}$ ). Then $g \circ f$ is also proper and inverse acyclic (with respect to $\mathscr{H}$ ).

Proof. $g \circ f$ is clearly proper. Take $z \in Z$ and let $A=g^{-1}(z)$. Then $A$ is an acyclic subset of $Y$. Let $f_{1}: f^{-1}(A) \rightarrow A$ be defined by $f$. Then $f_{1}$ is a map in $\mathscr{T}$ satisfying conditions (i) and (ii) for the Vietoris Theorem. Hence $f_{1^{*}}=\mathscr{C}\left(f^{-1}(A)\right) \rightarrow \mathscr{C}(A)$ is an isomorphism and $f^{-1}(A)=(g \circ f)^{-1}(z)$ is acyclic.

Theorem 5.2. (Eilenberg and Montgomery). Let $X$ be a compact metric space and $P$ a finite polyhedron. Let $f, g: X \rightarrow P$ be continuous maps such that $f$ is inverse acyclic. Then $\Lambda\left(g_{*} \circ f_{*}^{-1}\right)$ is defined and if $\Lambda\left(g_{*}^{\circ} f_{*}^{-1}\right) \neq 0$, then $f$ and $g$ have a coincidence. (See Theorem 4, [2].)

TheOREM 5.3. Finite polyhedra are M-Lefschetz spaces (relative to $\left.\mathscr{T}_{M}\right)$.

Proof. Let $P$ be a finite polyhedron and $F: P \rightarrow P$ an u.s.c. admissible map. Let $G_{0}, \cdots, G_{n}$ be an admissible sequence for $F$, where $G_{i}: Y_{i} \rightarrow Y_{i+1}$ with $Y_{0}=Y_{n+1}=P$ and all $Y_{i}$ are metric spaces. Then $G_{n^{*}} \circ \cdots \circ G_{0^{*}}$ has finite type since $P$ is finite.

Now suppose that $\Lambda\left(G_{n^{*}} \circ \cdots \circ G_{0^{*}}\right) \neq 0$. We prove that $F$ has a fixed point for the case $n=1$. The proof for arbitrary $n$ is an exact generalization of this proof. We have $G_{0}: P \rightarrow Y$ and $G_{1}: Y \rightarrow P$, where $Y$ is a metric space and $F=G_{1} \circ G_{0}$. Let $p_{0}, q_{0}$ be the projections associated with $G_{0}$. Then $p_{0}$ is inverse acyclic. The composition $G_{1} \circ q_{0}: \Gamma G_{0} \rightarrow P$ is an u.s.c. acyclic map.

Let $p_{1}, q_{1}$ be the projections associated with $G_{1} \circ q_{0}$; then $p_{1}$ is inverse acyclic. Then by (5.1) $p_{0} \circ p_{1}$ is inverse acyclic and we have $q_{1}, p_{0} \circ p_{1}: \Gamma G_{1} \circ q_{0} \rightarrow P$ where $\Gamma G_{1} \circ q_{0}$ is a compact metric space (since $P$ is finite). It is easy to check that $G_{1^{*}} \circ G_{0^{*}}=\dot{q}_{1^{*}} \circ\left(p_{0} \circ p_{1}\right)_{*}^{-1}$ and hence $\Lambda\left(q_{1^{*}} \circ\left(p_{0} \circ p_{1}\right)_{*}^{-1}\right) \neq 0$. Then by (5.2) $q_{1}$ and $p_{0} \circ p_{1}$ have a coincidence, say $\left(x, y, x^{\prime}\right) \in \Gamma G_{1} \circ q_{0}$. Since $\left(x, y, x^{\prime}\right)$ is a coincidence point for these maps, $x=x^{\prime}$. And $\left(x, y, x^{\prime}\right) \in \Gamma G_{1} \circ q_{0}$ implies that $x^{\prime}=x \in G_{1} \circ q_{0}(x, y)=$ $G_{1}(y)$ and $y \in G_{0}(x)$. Thus $x \in G_{1} \circ G_{0}(x)=F(x)$. 


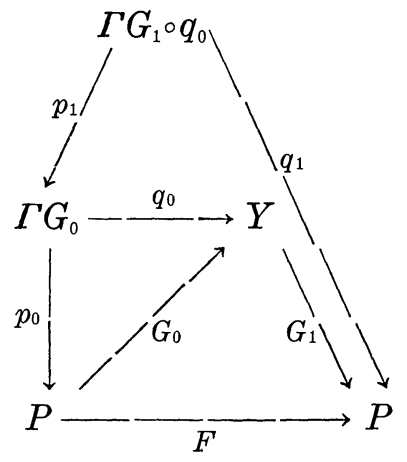

THEOREM 5.4. Every polyhedron with the Whitehead topology ([5], p.99) is an MA-space (relative to $\mathscr{T}_{M}$ ).

Proof. Let $P$ be a polyhedron with the Whitehead topology and $F: P \rightarrow P$ an u.s.c. compact admissible map. Let $G_{0}, \cdots, G_{n}$ be an admissible sequence for $F$. As in the proof of (5.3) it suffices to consider $n=1$. Then we have u.s.c. and acyclic maps $G_{0}: P \rightarrow Y$ and $G_{1}: Y \rightarrow P$ where $Y$ is a metric space and $F=G_{1} \circ G_{0}$.

Since $P$ has the Whitehead topology and $\overline{F(P)}$ is compact, there is a finite subpolyhedron $P^{\prime}$ of $P$ with $F(P) \subset P^{\prime}$. Let $Y^{\prime}=G_{0}\left(P^{\prime}\right)$ and define $F^{\prime}: P^{\prime} \rightarrow P^{\prime}, G_{0}^{\prime}: P^{\prime} \rightarrow Y^{\prime}, G_{1}^{\prime}: Y^{\prime} \rightarrow P^{\prime}$ by $F, G_{0}, G_{1}$. Consider the commmutative diagram where $G_{0}^{\prime \prime}, G_{1}^{\prime \prime}$ are defined by $G_{0}, G_{1}$ and the single-valued maps are inclusions.

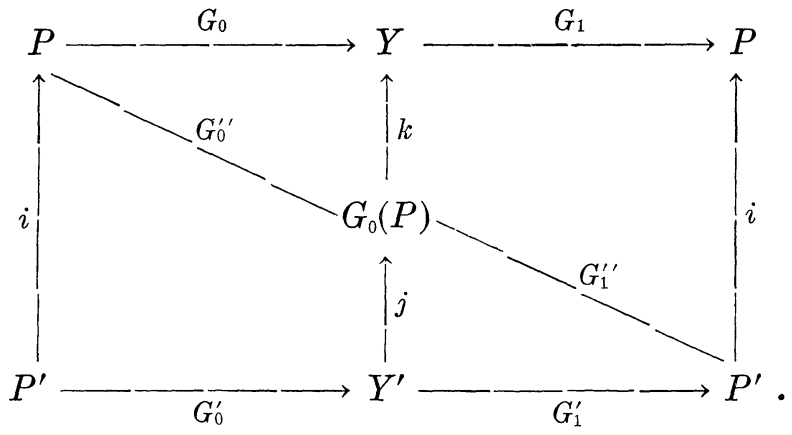

Then $G_{1^{*}}^{\prime} G_{0^{*}}^{\prime}=\left(G_{1^{*}}^{\prime \prime} G_{0^{*}}^{\prime \prime}\right) \circ i_{*}$ and $G_{1^{*} \circ} G_{0^{*}}=i_{*^{\circ}} \circ\left(G_{1^{*}}^{\prime \prime} G_{0^{*}}^{\prime \prime}\right)$. Since $\Lambda\left(G_{1^{*}}^{\prime} G_{0^{*}}^{\prime}\right)$ exists, (2.5) implies that $\Lambda\left(G_{1^{*}} G_{0^{*}}\right)$ exists and they are equal. Finally, if $\Lambda\left(G_{1^{*}} \circ G_{0^{*}}\right) \neq 0$, then by (5.3) $F^{\prime}$ has a fixed point. This is also fixed point for $F$.

THEOREM 5.5. (a) Every (metric) ANR is an MA-space (relative to $\left.\mathscr{T}_{M}\right)$. (b) Every compact (metric) ANR is an M-Lefschetz space (relative to $\mathscr{T}_{M}$ ). 
Proof. (a) Let $X$ be a (metric) $A N R$ and $\alpha \in \operatorname{Cov} X$. Then there is a polyhedron $P_{\alpha}$ (with the Whitehead topology) and continuous maps $h_{\alpha}: X \rightarrow P_{\alpha}, k_{\alpha}: P_{\alpha} \rightarrow X$ such that $k_{\alpha} \circ h_{\alpha}$ and $1_{X}$ are $\alpha$ homotopic. (See 1, Theorem 14.3.) In particular, $k_{\alpha^{*}} h_{\alpha^{*}}=1_{\mathscr{P}(X)}$ and $k_{\alpha} \circ h_{\alpha}$ and $1_{X}$ are $\alpha$-near. Then by (4.2) and (5.4) $X$ is an $M A$-space.

(b) The proof follows as in (a) using finite polyhedra and (5.3).

As a final application, the results (6.1) and (6.2) of [8] can be strengthened.

THEOREM 5.6. Let $X$ be any convex subset of a Banach space and $F: X \rightarrow X$ a compact u.s.c. map which is admissible (relative to $\left.\mathscr{T}_{M}\right)$. Then $F$ has a fixed point.

Proof. $X$ is an $A R$ and hence is an $M \Lambda$-space. Let $G_{0}, \cdots, G_{n}$ be an admissible sequence for $F$, where $G_{i}: Y_{i} \rightarrow Y_{i+1}$ with $Y_{0}=$ $Y_{n+1}=X$ and all $Y_{i}$ are metric spaces. It suffices to show

$$
\Lambda\left(G_{n^{*}} \circ \cdots \circ G_{0^{*}}\right) \neq 0 \text {. }
$$

But since $X$ is convex, the Lefschetz number of $G_{n^{*}} \circ \cdots \circ G_{0}^{*}$ is simply its trace in dimension 0 .

Take a point $y_{0} \in Y_{0}$ and for $i=0, \cdots, n$ let $y_{i+1}$ be an element of $G_{i}\left(y_{i}\right)$. Consider the diagram.

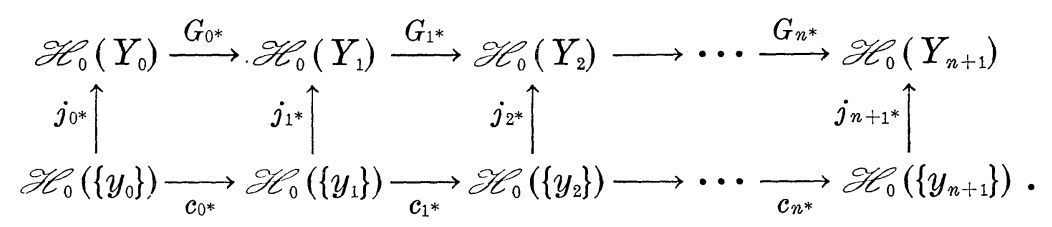

For each $i, j_{i+1} \circ c_{i}\left(y_{i}\right)=y_{i+1} \in G_{i}\left(y_{i}\right)=G_{i} \circ j_{i}\left(y_{i}\right)$. Thus $j_{i+1} \circ c_{i}$ is a crosssection of $G_{i} j_{i}$. But in general, if $F$ and $F^{\prime}$ are acyclic maps and $F$ is a cross-section of $F^{\prime}$, then $F_{*}=F_{*}^{\prime}$ (see [8] (3.7)). Hence the diagram commutes. Since $j_{0^{*}}$ and $j_{n+1^{*}}$ are isomorphisms, we conclude that $G_{n^{*}} \cdots G_{0^{*}}$ is an isomorphism and hence its Lefschetz number is nonzero.

Corollary 5.7. Let $X$ be a metric $A R$ and $F: X \rightarrow X$ a compact u.s.c. map which is admissible (relative to $\mathscr{T}_{M}$ ). Then $F$ has a fixed point.

Proof. $X$ can be imbedded as a closed subset of a convex set $C$ in a Banach space and there is a retraction $r: C \rightarrow X$. Then $F \circ r$ is a compact u.s.c. map and is admissible (relative to $\mathscr{T}_{M}$ ). By (5.8) $F \circ r$ has a fixed point. This must also be a fixed point for $F$. 


\section{REFERENCES}

1. J. Dugundji, Absolute neighborhood retracts and local connectedness in arbitrary metric spaces, Comp. Math., 13 (1958), 229-246.

2. S. Eilenberg and D. Montgomery, Fixed point theorems for multi-valued transformations, Amer. J. Math., 58 (1946), 214-222.

3. A. Granas, Generalizing the Hopf-Lefschetz fixed point theorem for non-compact $A N R$ 's, Proc. Symp. on Infinite Dimensional Topology, Baton Rouge, 1967.

4. A. Granas and L. Gorniewicz, Fixed point theorems for multi-valued mappings of the absolute neighborhood retracts, J. de Math. Pure et Appl. 49 (1970), 381-395.

5. S.-T. Hu, Theory of retracts, Wayne State Univ. Press, Detroit, 1965.

6. J. Jaworowski, and M. Powers, 1-spaces and fixed point theorems, Fund. Math., 64 (1969), 157-162.

7. J. Leray, Theorie des points fixes: Indice total et nombre de Lefschetz, Bull. Soc. Math. France, 87 (1959), 221-233.

8. M. Powers, Multi-valued mappings and Lefschetz fixed point theorems, Proc. Camb. Phil. Soc., 68 (1970), 619-630.

Received January 5, 1971 and in revised form July 12, 1971.

NoRThern ILlinoIS UNIVERSITY 


\section{PACIFIC JOURNAL OF MATHEMATICS}

\section{EDITORS}

H. SAMELSON

Stanford University

Stanford, California 94305

C. R. HobBY

University of Washington

Seattle, Washington 98105
J. DUGUNDJI

Department of Mathematics

University of Southern California

Los Angeles, California 90007

RICHARD ARENS

University of California

Los Angeles, California 90024

\section{ASSOCIATE EDITORS}

E. F. BECKENBACH

B. H. NeumanN

F WoLF

K. YoshidA

\section{SUPPORTING INSTITUTIONS}

UNIVERSITY OF BRITISH COLUMBIA

UNIVERSITY OF SOUTHERN CALIFORNIA

CALIFORNIA INSTITUTE OF TECHNOLOGY

UNIVERSITY OF CALIFORNIA

MONTANA STATE UNIVERSITY

STANFORD UNIVERSITY

UNIVERSITY OF NEVADA

NEW MEXICO STATE UNIVERSITY

OREGON STATE UNIVERSITY

UNIVERSITY OF OREGON

OSAKA UNIVERSITY

UNIVERSITY OF TOKYO

UNIVERSITY OF UTAH

WASHINGTON STATE UNIVERSITY

UNIVERSITY OF WASHINGTON

$\stackrel{*}{*} \stackrel{*}{*} \stackrel{*}{*}{ }^{*}{ }^{*}$ MMEICAN MATHEMATICAL SOCIETY

NAVAL WEAPONS CENTER

Printed in Japan by International Academic Printing Co., Ltd., Tokyo, Japan 


\section{Pacific Journal of Mathematics}

\section{Vol. 42, No. $1 \quad$ January, 1972}

Tage Bai Andersen, On Banach space valued extensions from split faces ........

David Marion Arnold, A duality for quotient divisible abelian groups of finite

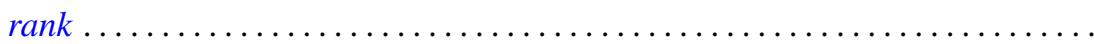

Donald Pollard Ballou, Shock sets for first order nonlinear hyperbolic

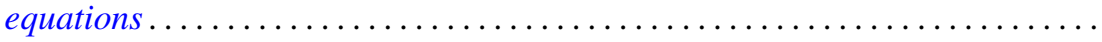

Leon Brown and Lowell J. Hansen, On the range sets of $H^{p}$ functions .........

Alexander Munro Davie and Arne Stray, Interpolation sets for analytic

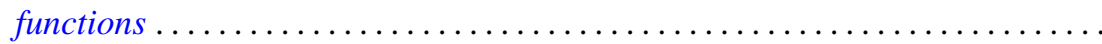

M. G. Deshpande, Structure of right subdirectly irreducible rings. II . . . . . . . . .

Barry J. Gardner, Some closure properties for torsion classes of abelian

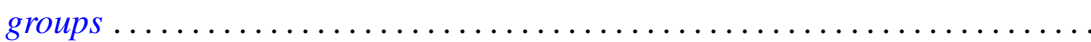

Paul Daniel Hill, Primary groups whose subgroups of smaller cardinality are

direct sums of cyclic groups . . . . . . . . . . . . . . . . . . .

Richard Allan Holzsager, When certain natural maps are equivalences .........

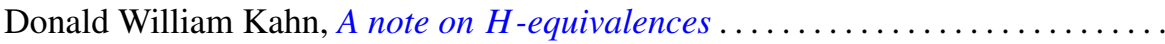

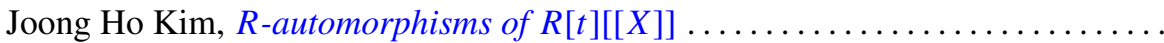

Shin'ichi Kinoshita, On elementary ideals of polyhedra in the 3-sphere.........

Andrew T. Kitchen, Watts cohomology and separability...

Vadim Komkov, A technique for the detection of oscillation of second order

ordinary differential equations .

Charles Philip Lanski and Susan Montgomery, Lie structure of prime rings of characteristic 2

Andrew Lenard, Some remarks on large Toeplitz determinants . .

Kathleen B. Levitz, A characterization of general Z.P.I.-rings. II .

Donald A. Lutz, On the reduction of rank of linear differential systems

David G. Mead, Determinantal ideals, identities, and the Wronskian ...

Arunava Mukherjea, A remark on Tonelli's theorem on integration in product

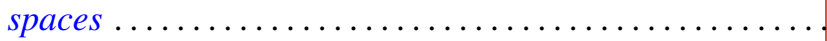

Hyo Chul Myung, A generalization of the prime radical in nonassociative rings.

John Piepenbrink, Rellich densities and an application to unconditionally nonoscillatory elliptic equations.

Michael J. Powers, Lefschetz fixed point theorems for a new class of multi-valued maps .

Aribindi Satyanarayan Rao, On the absolute matrix summability of a Fourier

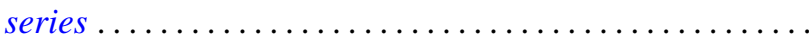

T. S. Ravisankar, On Malcev algebras ......................... 227

William Henry Ruckle, Topologies on sequences spaces . . . . . . . . . . . . . 235

Robert C. Shock, Polynomial rings over finite dimensional rings . . . . . . . . . 251

Richard Tangeman, Strong heredity in radical classes . . . . . . . . . . . . . . 259

B. R. Wenner, Finite-dimensional properties of infinite-dimensional spaces . . . . 267 